\title{
The Structure of the BLR in 3C 390.3
}

\author{
L. S. Nazarova ${ }^{1}$, N. G. Bochkarev ${ }^{1}$, and C. M. Gaskell ${ }^{2}$ \\ ${ }^{1}$ Euro-Asian Astronomical Society, Universitetskij Pr. 13, Moscow, 119992, Russia \\ Email: Isnazarova@rambler.ru, boch@sai.msu.ru \\ ${ }^{2}$ Astronomy Department, University of Texas, Austin, TX 78712-0259, USA \\ Email: gaskell@astro.as .utexas.edu
}

\begin{abstract}
Velocity-dependent flux ratios of the broad Ly $\alpha, \mathrm{C}$ IV, $\mathrm{H} \beta$, and $\mathrm{H} \alpha$ lines are used to investigate conditions in the archetypical displaced BLR peak emitter 3C 390.3. Our results suggest that gas producing the the UV emission lines has a lower density than the higher-velocity gas producing broad disk-like profile and is less flattened.
\end{abstract}

Keywords. galaxies: active, quasars: emission lines

The profiles of the broad emission lines in the majority of AGNs show a single peak close to the systemic velocity. 3C 390.3 is the archetype of a subclass of AGNs in which the Balmer lines show additional displaced peaks in their high-velocity wings. The highvelocity wings of these line profiles can be interpreted as emission from asymmetric turbulent disks. To attempt to understand the nature of the broad-line emission in 3C 390.3 we have constructed $\mathrm{CIV} / \mathrm{L} \alpha, \mathrm{L} \alpha / \mathrm{H} \beta$ and $\mathrm{H} \alpha / \mathrm{H} \beta$ flux ratios as a function of velocity for 3C 390.3 for "high" and "low" states of the ionizing continuum. We have used the photoionization code CLOUDY (Ferland et al. 1998) to attempt to understand the differences in physical conditions in emitting regions (see Nazarova et al. 2004, Gaskell et al. 2007, and Snedden \& Gaskell 2007 for details). We have taken approximate line-emitting region sizes from reverberation mapping by Dietrich et al. (1998), O'Brien et al. (1998), and Sergeev et al. (2002). Comparison of observed ratios with the models suggests that:

1)The increase in the $\mathrm{CIV} / \mathrm{L} \alpha$ ratio from the line centre to the wings can be explained by the density increasing from $10^{7-8} \mathrm{~cm}^{-3}$ at low velocity to $\sim 10^{9} \mathrm{~cm}^{-3}$ in the wings.

2) The increase in $\mathrm{L} \alpha / \mathrm{H} \beta$ and $\mathrm{H} \alpha / \mathrm{H} \beta$ from the wings to the core can be explained by a decrease in density from $10^{12-13} \mathrm{~cm}^{-3}$ for the high velocity gas to $10^{10-12} \mathrm{~cm}^{-3}$ towards the line core.

The difference in densities inferred for the high- and low-ionization gas at high velocities suggests that the two regions have somewhat different vertical locations. We have suggested (Nazarova et al. 2004) that high-ionization gas had a less flattened distribution than the higher-density, lower-ionization gas in the disk.

We are grateful for support from RFBR grant 09-02-01136 (LSN and NGB) and NSF grants AST-0307912 and AST-0803883 (CMG).

\section{References}

Dietrich, M., et al. 1998, ApJS, 115, 185

Ferland, G. J., et al. 1998, PASP, 110, 761.

Gaskell, C. M., Klimek, E. S., \& Nazarova, L. S. 2007, submitted to ApJ (arXiv:0711.1025)

Nazarova, L. S., Bochkarev, N. G., \& Gaskell, C. M. 2004, Astron. Astrophys. Trans., 23, 343

O'Brien, P. T., et al. 1998, ApJ, 509, 163

Sergeev, S. G., Pronik, V. I., Peterson, B. M., Sergeeva, E. A., \& Zheng, W. 2002, ApJ, 576, 660

Snedden, S. A. \& Gaskell, C. M. 2007, ApJ, 669, 126 